\title{
Microfragmented Adipose Tissue With Adjuvant Platelet-Rich Plasma Combination Therapy for Partial-Thickness Supraspinatus Tear
}

\author{
Anuj Marathe ${ }^{1}$, Bo Song ${ }^{1}$, Prathap Jayaram ${ }^{1}$ \\ 1. H. Ben Taub Department of Physical Medicine and Rehabilitation, Baylor College of Medicine, Houston, USA
}

Corresponding author: Anuj Marathe, anuj.marathe21@gmail.com

\begin{abstract}
A 50-year-old male presented with acute, sharp, right shoulder pain. Ultrasound of the right shoulder revealed a partial thickness tear of the supraspinatus. After conservative management failed to provide any relief, he was treated with microfragmented adipose tissue (MFAT) injection followed by platelet-rich plasma (PRP) at 14 weeks. At the 28 -week follow-up, he showed significant improvement in pain and mobility with a resolution of the tear on ultrasound. While PRP has been shown to confer some protection against retears, very few studies have investigated the efficacy of MFAT use in rotator cuff pathology. In this case, we used a combination of MFAT and PRP to successfully treat a partial thickness supraspinatus tear. These agents may function in a synergistic manner, with MFAT providing a cell scaffold and PRP modulating the cellular environment to optimize healing. Further studies are needed to better understand the mechanism of this treatment modality in treating similar conditions.
\end{abstract}

Review began 06/04/2021 Review ended 06/07/2021 Published 06/10/2021

๑) Copyright 2021

Marathe et al. This is an open access article distributed under the terms of the Creative Commons Attribution License CC-BY 4.0., which permits unrestricted use, distribution, and reproduction in any medium, provided the original author and source are credited.
Categories: Physical Medicine \& Rehabilitation

Keywords: microfragmented adipose tissue, platelet-rich plasma, supraspinatus, sports medicine, regenerative medicine

\section{Introduction}

Rotator cuff tears affect an estimated $25 \%$ of individuals in their $60 \mathrm{~s}$ and $50 \%$ of individuals in their 80 s [1]. The pathophysiology is multifactorial and includes excess mechanical loads, intrinsic joint anatomy, muscle overuse, and age-related degeneration [2]. Recent studies have shown postsurgical retear rates as high as $13.1 \%$ for full-thickness tears, with $40 \%$ of asymptomatic partial-thickness tears eventually progressing to full-thickness tears $[3,4]$. As an alternative to surgical intervention, minimally invasive regenerative strategies such as microfragmented adipose tissue (MFAT) and platelet-rich plasma (PRP) have recently gained popularity [5]. This case highlights the treatment of a partial-thickness supraspinatus tear with combination MFAT and PRP therapy.

\section{Case Presentation}

A 50-year-old healthy male with no significant past medical history presented with acute, sharp, right shoulder pain after reaching behind his car seat. On examination, he exhibited significant right shoulder hiking with $8 / 10$ pain on the visual analog scale (VAS) at $70^{\circ}$ of abduction and $45^{\circ}$ external rotation with limited range of motion (ROM) and weakness when reaching overhead. He also had 4/5 strength during shoulder abduction with positive Neer's and Hawkin's tests.

Ultrasound of the right shoulder revealed a partial-thickness supraspinatus tear measuring $2.5 \mathrm{~mm} \times 6.5 \mathrm{~mm}$ (Figure 1A). After little relief following six weeks of physical therapy focusing on rotator cuff and shoulder strengthening and stretching, he opted for a combination regenerative strategy using MFAT and a subsequent PRP injection. For the MFAT injection, fat was harvested from bilateral gluteal areas and $60 \mathrm{~mL}$ of lipoaspirate was collected. The sample was processed through a Lipogems ${ }^{\circledR}$ device (Lipogems International, Milan, Italy), and $3 \mathrm{~mL}$ was injected directly into the supraspinatus tendon tear site. He was also restarted on a rotator cuff rehabilitation program one-week post-procedure for 12 weeks. Follow-up at 14 weeks showed moderately improved ROM and pain reduction. At this time, a subsequent PRP injection (5 $\mathrm{mL}$ total) was performed into the supraspinatus tear site to further augment the healing process. Follow-up at 28 weeks showed near full recovery of pain (1/10 on VAS) and ROM with complete healing of the supraspinatus tear on ultrasound (Figure 1B). 


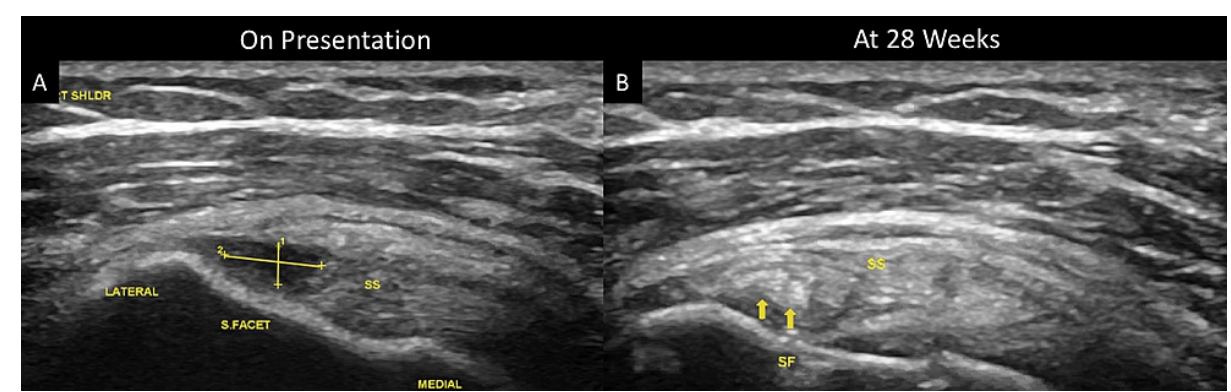

FIGURE 1: Ultrasound on presentation (A) and at 28 weeks (B).

(A) Supraspinatus partial-thickness tear near tendon insertion site measuring $2.5 \mathrm{~mm} \times 6.5 \mathrm{~mm}$. (B) Resolved supraspinatus tear.

\section{Discussion}

Mesenchymal stem cells (MSCs) have recently gained traction for their potential applications in regenerative medicine. These cells can be extracted from bone marrow or fat and are capable of multilineage differentiation [6]. Traditionally, adipose tissue is enzymatically digested into a stromal vascular fraction (SVF) containing adipose MSCs; however, MFAT uses mild mechanical forces to break up the tissue which preserves the microvascular stroma [7]. Vezzani et al. found that compared to SVF, MFAT has much greater regenerative potential through increased production of cytokines and growth factors responsible for angiogenesis and tissue repair [8]. This is thought to be a result of the intact microvasculature and stromal tissue that allows for better cell scaffolding and long-term MSC survival as well as increased recruitment of CD-163+ wound-healing macrophages compared to SVF [9].

MFAT use has shown promising results in treating knee osteoarthritis (KOA). In fact, it was shown to increase glycosaminoglycan content in existing cartilage even in patients with grade III and IV KOA [10]. Mautner et al. conducted a prospective analysis on patients who received MFAT injections for KOA and found a significant improvement in function and pain [11]. Russo et al. showed similar results in patients with degenerative chondral lesions in the knee who had failed conservative treatment (rehabilitation and other injectables) for at least 12 months [12].

However, there is a severe paucity of clinical data for MFAT use in rotator cuff pathologies. One retrospective animal study examined gait data and tendon ultrasounds of dogs with supraspinatus tendinopathy treated with MFAT plus PRP injection and found significant improvements in gait function and tendon inflammation after 90 days [13]. One human case series by Striano et al. assessed the efficacy of MFAT in rotator cuff tears and shoulder osteoarthritis [14]. Using ultrasound guidance, they directly injected MFAT into tears as well as into the glenohumeral joint space and found statistically significant improvements in pain and function in the short and long term [14]. Additionally, Cherian et al. described one case where MFAT treatment provided complete pain relief for a patient with chronic rotator cuff tear after conservative management had failed [15].

Similar to MFAT, PRP is another biologic agent being studied for its regenerative potential. Platelet alphagranules contain high levels of growth factors such as platelet-derived growth factor, transforming growth factor-beta, and vascular endothelial growth factor which are believed to help with physiologic wound healing at injected sites [16]. In addition, arthroscopic rotator cuff repairs that used adjuvant PRP had lower retear rates and improved clinical outcomes [17]. Cai et al. also found improved pain and function in patients with small-to-medium partial-thickness rotator cuff tears treated with PRP [18]. Finally, a recent systematic review found significantly lower rotator cuff retear rates in patients treated with PRP [19]. While they also found statistical significance between PRP and placebo in functional scores, they noted that it did not meet the minimum clinical significance criteria [19].

\section{Conclusions}

This case demonstrates the first use of MFAT and PRP combination therapy in a human to treat a partialthickness supraspinatus tear. Given the mechanisms of both modalities, we suspect a synergistic mechanism. It is likely that the MFAT filled in the torn region, laid a scaffold, and began the repair process, after which PRP further accelerated healing. Surprisingly, the potential synergistic effects of these agents are yet to be studied. Future studies could use standard patient-reported outcome metrics to directly compare efficacy to the current standards of care. While our report is limited to one case, the promising results suggest that utilizing both biologics in combination may lead to better outcomes, especially in rotator cuff tears.

\section{Additional Information}




\section{Disclosures}

Human subjects: Consent was obtained or waived by all participants in this study. Conflicts of interest: In compliance with the ICMJE uniform disclosure form, all authors declare the following: Payment/services info: All authors have declared that no financial support was received from any organization for the submitted work. Financial relationships: All authors have declared that they have no financial relationships at present or within the previous three years with any organizations that might have an interest in the submitted work. Other relationships: All authors have declared that there are no other relationships or activities that could appear to have influenced the submitted work.

\section{References}

1. Yamamoto A, Takagishi K, Osawa T, Yanagawa T, Nakajima D, Shitara H, Kobayashi T: Prevalence and risk factors of a rotator cuff tear in the general population. J Shoulder Elbow Surg. 2010, 19:116-20. 10.1016/j.jse.2009.04.006

2. Via AG, De Cupis M, Spoliti M, Oliva F: Clinical and biological aspects of rotator cuff tears . Muscles Ligaments Tendons J. 2013, 3:70-9. 10.11138/mltj/2013.3.2.070

3. Kim IB, Kim MW: Risk factors for retear after arthroscopic repair of full-thickness rotator cuff tears using the suture bridge technique: classification system. Arthroscopy. 2016, 32:2191-200. 10.1016/j.arthro.2016.03.012

4. Mall NA, Kim HM, Keener JD, et al.: Symptomatic progression of asymptomatic rotator cuff tears: a prospective study of clinical and sonographic variables. J Bone Joint Surg Am. 2010, 92:2623-33. 10.2106/JBJS.I.00506

5. Charles MD, Christian DR, Cole BJ: The role of biologic therapy in rotator cuff tears and repairs . Curr Rev Musculoskelet Med. 2018, 11:150-61. 10.1007/s12178-018-9469-0

6. Han Y, Li X, Zhang Y, Han Y, Chang F, Ding J: Mesenchymal stem cells for regenerative medicine. Cells. 2019, 8:886. 10.3390/cells8080886

7. Bianchi F, Maioli M, Leonardi E, et al.: A new nonenzymatic method and device to obtain a fat tissue derivative highly enriched in pericyte-like elements by mild mechanical forces from human lipoaspirates. Cell Transplant. 2013, 22:2063-77. 10.3727/096368912X657855

8. Vezzani B, Shaw I, Lesme H, Yong L, Khan N, Tremolada C, Péault B: Higher pericyte content and secretory activity of microfragmented human adipose tissue compared to enzymatically derived stromal vascular fraction. Stem Cells Transl Med. 2018, 7:876-86. 10.1002/sctm.18-0051

9. Desando G, Bartolotti I, Martini L, et al.: Regenerative features of adipose tissue for osteoarthritis treatment in a rabbit model: enzymatic digestion versus mechanical disruption. Int J Mol Sci. 2019, 20:2636. 10.3390/ijms20112636

10. Hudetz D, Borić I, Rod E, et al.: The effect of intra-articular injection of autologous microfragmented fat tissue on proteoglycan synthesis in patients with knee osteoarthritis. Genes (Basel). 2017, 8:270. 10.3390/genes8100270

11. Mautner K, Bowers R, Easley K, Fausel Z, Robinson R: Functional outcomes following microfragmented adipose tissue versus bone marrow aspirate concentrate injections for symptomatic knee osteoarthritis. Stem Cells Transl Med. 2019, 8:1149-56. 10.1002/sctm.18-0285

12. Russo A, Condello V, Madonna V, Guerriero M, Zorzi C: Autologous and micro-fragmented adipose tissue for the treatment of diffuse degenerative knee osteoarthritis. J Exp Orthop. 2017, 4:33. 10.1186/s40634-0170108-2

13. Canapp SO Jr, Canapp DA, Ibrahim V, Carr BJ, Cox C, Barrett JG: The use of adipose-derived progenitor cells and platelet-rich plasma combination for the treatment of supraspinatus tendinopathy in 55 dogs: a retrospective study. Front Vet Sci. 2016, 3:61. 10.3389/fvets.2016.00061

14. Striano RD, Malanga GA, Bilbool N, Azatullah K: Refractory shoulder pain with osteoarthritis, and rotator cuff tear, treated with micro-fragmented adipose tissue. Orthop Spine Sports Med. 2018, 2:014.

15. Cherian C, Malanga GA, Hogaboom N, Pollack MA, Dyson-Hudson TA: Autologous, micro-fragmented adipose tissue as a treatment for chronic shoulder pain in a wheelchair using individual with spinal cord injury: a case report. Spinal Cord Ser Cases. 2019, 5:46. 10.1038/s41394-019-0186-8

16. Wu PI, Diaz R, Borg-Stein J: Platelet-rich plasma. Phys Med Rehabil Clin N Am. 2016, 27:825-53. 10.1016/j.pmr.2016.06.002

17. Han C, Na Y, Zhu Y, Kong L, Eerdun T, Yang X, Ren Y: Is platelet-rich plasma an ideal biomaterial for arthroscopic rotator cuff repair? A systematic review and meta-analysis of randomized controlled trials. J Orthop Surg Res. 2019, 14:183. 10.1186/s13018-019-1207-9

18. Cai YU, Sun Z, Liao B, Song Z, Xiao T, Zhu P: Sodium hyaluronate and platelet-rich plasma for partialthickness rotator cuff tears. Med Sci Sports Exerc. 2019, 51:227-33. 10.1249/MSS.0000000000001781

19. Chen X, Jones IA, Togashi R, Park C, Vangsness CT Jr: Use of platelet-rich plasma for the improvement of pain and function in rotator cuff tears: a systematic review and meta-analysis with bias assessment. Am J Sports Med. 2020, 48:2028-41. 10.1177/0363546519881423 